\title{
Optimized design of harmonic-injection dividers
}

\author{
Franco Ramírez, Almudena Suárez
}

\author{
Communications Engineering Department, University of Cantabria, 39005, Spain
}

\begin{abstract}
A new formulation is presented for the efficient harmonic-balance analysis of the division bandwidth of frequency dividers by a high order. The procedure is based on some mathematical properties of the solution curve under low-amplitude of the input generator. Through a simple fitting technique, it is possible to determine the variation of the synchronization bandwidth versus any design parameter, while keeping constant the central frequency of the division band. The procedure also enables a prediction of the frequency-division interval for any value of the input-generator amplitude within the region of linear behavior with respect to the input source. It has been applied to the optimization of the input matching network in a frequency divider by 10 , which uses a nonlinear transmission line (NLTL) as a feedback network.
\end{abstract}

Index Terms - Harmonic-injection frequency divider, harmonic balance, synchronization.

\section{INTRODUCTION}

Frequency dividers are essential components of frequency synthesizers, widely used in radio communication systems. The harmonic-injection dividers can operate at high input frequencies and enable a versatile design with a small number of active devices and low power consumption [1,2]. However, the harmonic balance (HB) simulation of frequency dividers is demanding, so obtaining an optimized prototype can be a difficult task. In fact, $\mathrm{HB}$ must be combined with additional techniques to sustain the subharmonic oscillation [3,4], as otherwise it converges to a trivial solution, having the input frequency as fundamental. This undesired convergence can be prevented with the use of an auxiliary generator (AG) at the subharmonic frequency [3]. Setting the phase reference at the AG location, the bandwidth is obtained by sweeping the input generator phase from $0^{\circ}$ to $360^{\circ}[3,4]$. More harmonic terms will be required for higher division order $N$. In general, the lengthy simulations will prevent an efficient analysis of the impact of the circuit elements on the operation bandwidth and, thus, an optimized design.

In view of this difficulty, a new formulation of general application to harmonic-injection dividers has been derived in this work. It should be applied in combination with a suitable choice of the circuit topology, so as to enable harmonic-injection division at low input power, with the divider behaving linearly with respect to the input source. This methodology provides the variation of the division bandwidth versus any design parameter, while keeping constant the central operation frequency at each parameter value. Preserving the central frequency is a relevant property of the method since, by default, any change of a parameter value (a line length, bias voltage etc.) will give rise to a shift of the division band. Unlike previous techniques $[3,4]$, based on a numerical determination of an admittance-function derivatives about the free-running solution, in the new method the solution curve is fitted with a minimum number of injection-locked solution points, which should increase the accuracy and reduce the computational cost. A criterion is also provided to estimate the limit of the input-amplitude interval with linear behavior with respect to the input source.

\section{SiMULATION TECHNIQUE}

The simulation technique presented here for the first time has two goals: (1) maximization of the division bandwidth and (2) preservation of the central frequency of the operation band. Assuming linear behaviour with respect to the input source, this central frequency should correspond to the free-running frequency $\omega_{o}$ [3]. For an optimized design, the division bandwidth will be calculated versus one or more design parameters, selecting the values that provide the maximum bandwidth. Let the parameter $\eta$ be considered. At each sweep step $\eta_{i}$, the circuit is solved in two stages. In a first stage it operates in free-running regime. In a second stage, it operates in synchronized regime with respect to an input source with amplitude $E_{\text {in }}$ and frequency $\omega=N \omega_{0}$. The analysis is carried out with an AG [4]. The voltage AG, connected in parallel to a sensitive circuit node, such as the terminal nodes of transistor devices, must fulfil a non-perturbation condition, given by the zero value of the ratio between the current through this generator and the voltage delivered $Y_{A G}=0$ [4]. This condition is solved through optimization. At each parameter step $\left(\eta_{i}\right)$, the procedure is as follows. At Stage 1, the input amplitude is set to zero $E_{\text {in }}=0$ and the AG frequency is made constant at the desired freerunning oscillation frequency (center of the division band), that is $\omega_{A G}=\omega_{o}$. Then the AG amplitude (agreeing with the voltage amplitude at $\omega_{\mathrm{o}}$ at the connection node $V_{A G}=V_{o}$ ) and another circuit parameter, such as a bias voltage $V_{\text {bias }}$, are optimized in order to fulfil $Y_{A G}\left(V_{\text {bias }}, A_{A G}\right)=0$ at $\omega_{o}$. At Stage 2, the input source with low amplitude $E_{\text {in }}$ is introduced and two values of the input-generator phase $\left(\phi_{1}\right.$ and $\left.\phi_{2}\right)$ are considered, with the 
phase reference at the AG node. At each of the two phase values, the non-perturbation condition is solved in terms of the $\mathrm{AG}$ amplitude $A_{A G}$ and frequency $\omega_{A G}$, that is., $Y_{A G}\left(A_{A G}, \omega_{A G}\right)=0$.

In $[3,4]$, it was demonstrated that provided a suitable design is carried out, for small input amplitude, the divider solution curve versus $\omega / N$ can be approached with an ellipse. This is obtained linearizing the total admittance function $Y_{A G}$ about the free-running solution. Renaming $Y_{A G}$ as $Y$, the equation is $[3,4]$ :

$$
\begin{aligned}
& Y_{v}^{r}\left(V-V_{o}\right)+Y_{\omega}^{r}\left(\frac{\omega}{N}-\omega_{o}\right)=\frac{\partial Y^{r}}{\partial E_{\text {in }}^{r}} E_{\text {in }} \cos (\phi)+\frac{\partial Y^{r}}{\partial E_{\text {in }}^{i}} E_{\text {in }} \sin (\phi) \\
& Y_{v}^{i}\left(V-V_{o}\right)+Y_{\omega}^{i}\left(\frac{\omega}{N}-\omega_{o}\right)=\frac{\partial Y^{i}}{\partial E_{i n}^{r}} E_{\text {in }} \cos (\phi)+\frac{\partial Y^{i}}{\partial E_{\text {in }}^{i}} E_{\text {in }} \sin (\phi)
\end{aligned}
$$

where the superindexes indicate real and imaginary part, and the subindexes indicate the variable with respect to which the derivative is calculated. Solving for the frequency and amplitude increments, one obtains:

$$
\begin{aligned}
& \omega / N-\omega_{o}=\Delta \omega=E_{\text {in }} k_{w} \sin \left(\phi-\alpha_{v}\right) \\
& V_{\text {in }}-V_{o}=\Delta V=E_{\text {in }} k_{v} \sin \left(\phi-\alpha_{\omega}\right)
\end{aligned}
$$

where $k_{w}$ and $k_{v}$ are proportionality constants and $\alpha_{v}$ and $\alpha_{\omega}$ are constant phase shifts. Using the phase $\phi$ as an intermediate variable, the parameters in each of the two equations in (2), can be obtained from the two injectionlocked solutions calculated with the AG: $\left(\phi_{1}, \Delta V_{1}, \Delta \omega_{1}\right)$ and $\left(\phi_{2}, \Delta V_{2}, \Delta \omega_{2}\right)$. The expansion of (2)(a), denoting $c=\cos \alpha_{v}$ and $s=\sin \alpha_{v}$, provides the system:

$\Delta \omega_{1}=E_{\text {in }} k_{w} c \sin \phi_{1}-E_{\text {in }} k_{w} s \cos \phi_{1}=$

$=E_{\text {in }} c_{w} \sin \phi_{1}-E_{\text {in }} s_{w} \cos \phi_{1}$

$\Delta \omega_{2}=E_{\text {in }} c_{w} \sin \phi_{2}-E_{\text {in }} s_{w} \cos \phi_{2}$

where the new constant parameters $c_{w}=k_{w} c, s_{w}=k_{w} s$ have been defined. The unknowns to be calculated are $c_{w}$ and $s_{w}$, which are given by:

$c_{w}=\frac{-\Delta \omega_{1} \cos \phi_{2}+\Delta \omega_{2} \cos \phi_{1}}{E_{i n 1} \sin \left(\phi_{2}-\phi_{1}\right)}, s_{w}=\frac{-\Delta \omega_{1} \sin \phi_{2}+\Delta \omega_{2} \sin \phi_{1}}{E_{i n 1} \sin \left(\phi_{2}-\phi_{1}\right)}$

Then, the relationship between the frequency increment $\Delta \omega$ and the phase shift $\phi$ is:

$\Delta \omega(\phi)=E_{\text {in }}\left[c_{w} \sin (\phi)-s_{w} \cos (\phi)\right]=E_{\text {in }} k_{w} \sin \left(\phi-\alpha_{v}\right)$

So $k_{w}=\left(c_{w}^{2}+s_{w}^{2}\right)^{1 / 2}$ and $\alpha_{v}=\operatorname{atan}_{2}\left(s_{w} / c_{w}\right)$. From (4), at each $E_{i n}$, the edges of the synchronization bandwidth correspond to the phase values $\phi_{m}=-\pi / 2+\alpha_{v}$ and $\phi_{M}=\pi / 2+\alpha_{v}$. In the plane $\omega / N, E_{i n}$, the synchronization region is delimited by the two straight lines:

$E_{\text {in }}=\omega /\left(N k_{w}\right)-\omega_{o} / k_{w}, \quad E_{\text {in }}=-\omega /\left(N k_{w}\right)+\omega_{o} / k_{w}$

The output frequency bandwidth $B_{w}$ increases linearly with the input amplitude $\mathrm{E}_{\mathrm{in}}$, according to $B_{w}=2 E_{i n} k_{w}$, which will be fulfilled provided that the circuit behaves linearly with respect to $E_{i n}$. For a given circuit design, the limit of the linear region can be estimated from the deviation of the frequency $\omega$ obtained with the AG in HB for $\phi_{m}=-\pi / 2+\alpha_{v}$ and $\phi_{M}=\pi / 2+\alpha_{v}$ and the prediction by (6)

Proceeding in identical manner with the amplitude increment $\Delta V$, it is possible to obtain the following constant parameters:

$c_{v}=\frac{-\Delta V_{1} \cos \phi_{2}+\Delta V_{2} \cos \phi_{1}}{E_{i n 1} \sin \left(\phi_{2}-\phi_{1}\right)}, s_{v}=\frac{-\Delta V_{1} \sin \phi_{2}+\Delta V_{2} \sin \phi_{1}}{E_{i n 1} \sin \left(\phi_{2}-\phi_{1}\right)}$

And the relationship between the amplitude increment $\Delta V$ and the phase shift $\phi$ is:

$$
\Delta V(\phi)=E_{\text {in }}\left[c_{v} \sin (\phi)-s_{v} \cos (\phi)\right]=E_{\text {in }} k_{v} \sin \left(\phi-\alpha_{w}\right)
$$

The ellipse equation is directly obtained making the phase $\phi$ disappear from (5) and (8), which is done solving for $\sin (\phi)$ and $\cos (\phi)$ :

$$
\begin{aligned}
& k_{v}^{2} \Delta \omega^{2}+k_{w}^{2} \Delta V^{2}-2 k_{v} k_{\omega} \cos \left(\alpha_{\mathrm{w}}-\alpha_{\mathrm{v}}\right) \Delta V \Delta \omega= \\
& =E_{\text {in }}^{2}\left(k_{v} k_{\omega}\right)^{2} \sin ^{2}\left(\alpha_{\mathrm{w}}-\alpha_{\mathrm{v}}\right)
\end{aligned}
$$

As the free-running solution changes at each sweep step, the stability [5] of the optimum solution (the one with the broadest bandwidth) must be verified.

\section{APPLICATION TO A FREQUENCY DIVIDER BY 10}

As an example, the technique will be applied to the divider circuit in Fig. 1. It includes a nonlinear transmission line NLTL [6] in the feedback loop, acting as a multiplier by $N-1=9$, which enables the division by $N=10$. The circuit in Fig. 1 is similar to the nonoptimized one in [2], although the input network used here is constituted by a parallel inductor and a distributed impedance transformer.

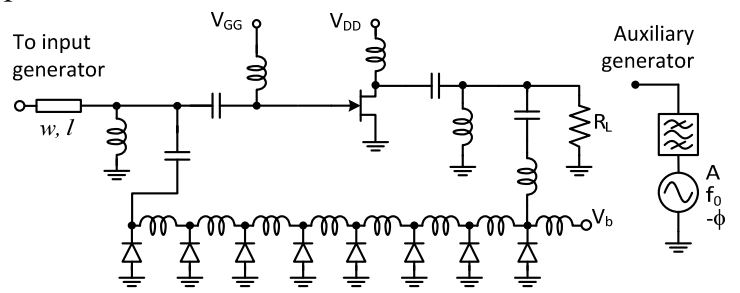

Fig.1 Frequency divider based on an NLTL. It uses an ATF33143 transistor and SMV1247 varactor diodes.

The collection of ellipses calculated with (9) for fixed input power $P_{\text {in }}=-10 \mathrm{dBm}$, increasing the width $w$ of the input transmission line, is represented in Fig. 2. At each analysis in free-running conditions, $A_{A G}$ and bias voltage $V_{\text {bias }}$ of the NLTL varactor diodes are optimized in order to fulfil $Y_{A G}=0$. Even though all these different designs exhibit the same free-running frequency $f_{o}=0.9 \mathrm{GHz}$, the free-running solution does change, which explains the variation of the tilt angle. The accuracy is validated in two cases $(w=0.15 \mathrm{~mm}$ and $w=2.65 \mathrm{~mm}$, corresponding to the line impedance $Z=29 \mathrm{Ohm})$, tracing the whole solution curve with HB (41 points per ellipse, instead of only two). The stability [5] of the initial and final solutions has been successfully verified. For each width 
value, the upper section of the ellipse, comprised between the two turning points is stable, and the lower section is unstable. The deviation from the perfect ellipse in (9) changes (slightly) with the parameter value. It is larger in the lower section of the ellipse, expected to exhibit more irregular shape when the circuit operates nonlinearly with respect to the input source. The bandwidth calculated with $B_{w}=2 E_{i n} k_{w}$ is traced versus $w$ in Fig. 3. Measurements are superimposed. The divider has been optimized since the technique has allowed increasing five times the original operation bandwidth. Furthermore, the central frequency of the band has been preserved, which would be impossible through a simple sweep or tuning technique. The measured spectrum is in Fig. 4.

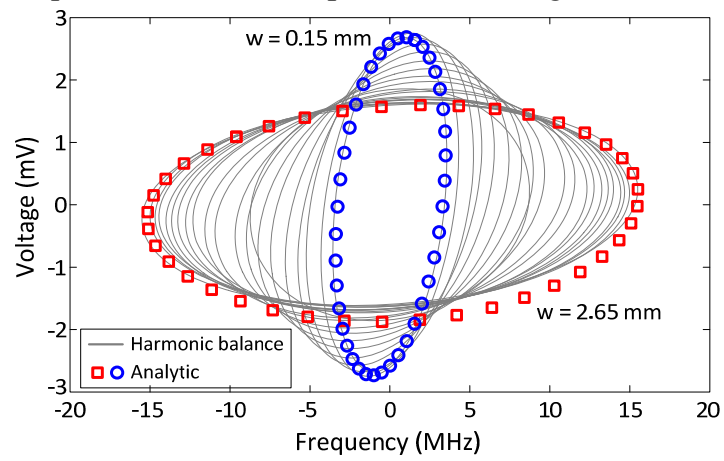

Fig. 2 Collection of ellipses identified with the new analysis procedure, validated with HB simulations.

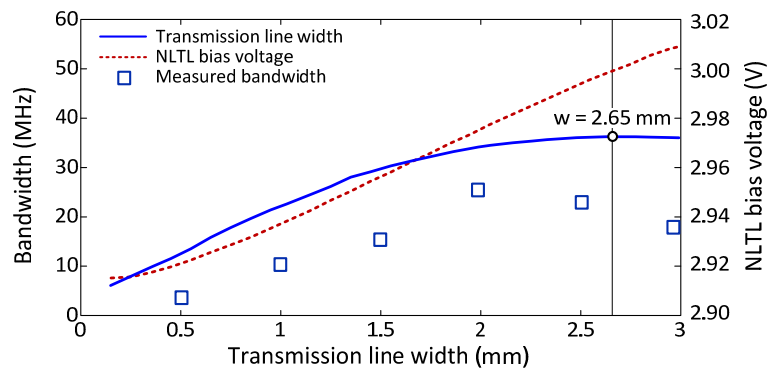

Fig. 3 Variation of the frequency-division bandwidth versus the transmission-line width at $P_{i n}=-10 \mathrm{dBm}$.

The synchronization region in the plane $\omega, E_{\text {in }}$ is shown in Fig. 5. The limit of the linear region has been estimated performing two HB analyses, at $\phi=\pi / 2+\alpha_{v}$ and $\phi=-$ $\pi / 2+\alpha_{v}$, for each $E_{\text {in }}$ value. Besides the deviation from a perfect ellipse, as $E_{\text {in }}$ increases, the bandwidth is no longer centred about $N f_{o}$. Projections of the full $\mathrm{HB}$ solution curve for two $E_{\text {in }}$ values are also presented. In this particular case, the capability to obtain a noticeable bandwidth is due to the novel topology, using the NLTL. The bandwidth of an ordinary oscillator injection-locked at the $10^{\text {th }}$ harmonic component would be negligible in most cases. Its observation might require input power that does not belong to the interval with linear behaviour with respect to the input source and the bandwidth might not be cantered about the free-running oscillation.

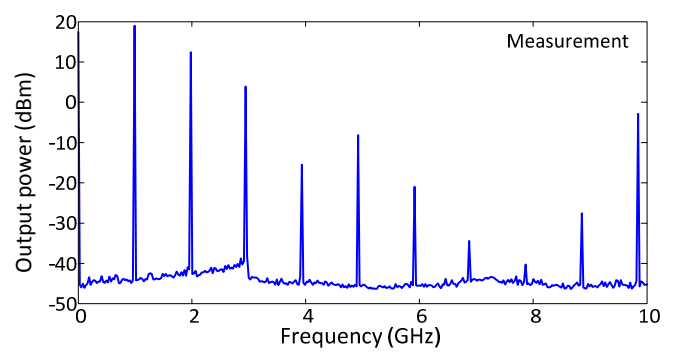

Fig. 4 Measured spectrum of the frequency-divider.

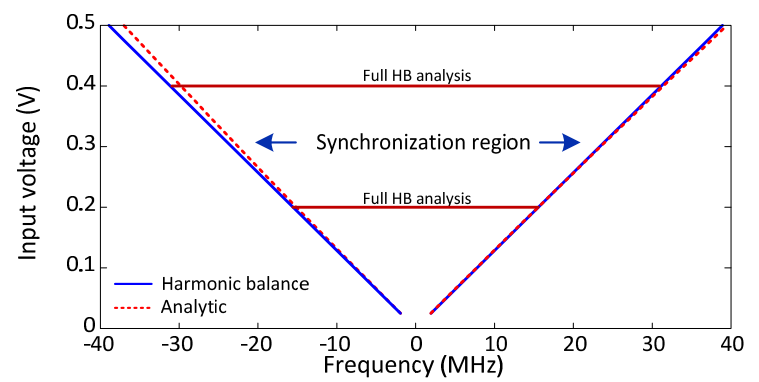

Fig. 5 Synchronization region in the plane $\omega, E_{i n}$. The limit of the linear region has been estimated performing two HB analyses at $\phi=\pi / 2+\alpha_{v}$ and $\phi=-\pi / 2+\alpha_{v}$, respectively.

\section{CONCLUSION}

A new formulation has been presented for the efficient analysis of the division bandwidth of frequency-dividers versus relevant design parameters. It is based on the identification of the solution curve using a small number of HB simulations. An advantage is the capability to maintain the central frequency of the division bandwidth under the variation of the particular analysis parameter.

\section{ACKNOWLEDGEMENT}

Spanish project TEC2011-29264-C03-01 for financial support.

\section{REFERENCES}

[1] S. Verma, H. R. Rategh, T. H. Lee, "A Unified Model for Injection-Locked Frequency Dividers," IEEE Jour. of Solidstate circuits, vol. 38, no. 6, Jun. 2003, pp. 1015-1027.

[2] F. Ramírez, A. Suárez, "Harmonic-injection divider based on feedback through a nonlinear transmission line", EuMIC 2013, Nuremberg, Oct., 2013.

[3] F. Ramírez, E. de Cos, A. Suárez, Nonlinear analysis tools for the optimized design of harmonic-injection frequency dividers, IEEE T-MTT, vol. 51, no. 6, pp. 1752-1762, 2003.

[4] A. Suárez, Analysis and Design of Autonomous Microwave Circuits, Hoboken, New Jersey: Wiley - IEEE Press, 2009.

[5] J. Jugo, J. Portilla, A. Anakabe, A. Suárez, and J. M. Collantes, "Closed-loop stability analysis of microwave amplifiers," IEE Electronics Letters, vol. 37, pp. 226-228, Feb. 2001.

[6] F. Martín, et al., Optimization of Nonlinear Transmission Lines for Harmonic Generation", International Journal of Infrared and Millimeter Waves, vol. 23, no. 1, pp 95-103, Jan. 2002. 